\title{
APACHE II score predicts mortality in patients requiring prolonged ventilation in a weaning center
}

\author{
Anna Rojek-Jarmuła1, 2, Rainer Hombach ${ }^{1}$, Łukasz J. Krzych ${ }^{3}$ \\ ${ }^{1}$ Weaning Station, Marienhaus Klinikum Eifel, Neuerburg, Germany \\ ${ }^{2}$ Department of Anaesthesiology and Intensive Care, Marienhaus Klinikum Eifel St. Elizabeth, \\ Gerolstein, Germany \\ ${ }^{3}$ Department of Cardiac Anaesthesiology and Intensive Care, Medical University of Silesia \\ in Katowice, Poland
}

\begin{abstract}
Background: Little is known about the diagnostic accuracy of the APACHE II scoring system in prolonged mechanical ventilation. The aim of this study was to assess the utility of APACHE II in order to predict in-hospital mortality, length of stay (LOS) and discharge destination of patients in a weaning centre.

Methods: The study group included 130 consecutive patients (median age 71 years; IQR 62-77). APACHE II was assessed based on the worst values taken during the first 24 hours post admission. The primary outcome was in-hospital mortality. LOS and discharge destination were considered secondary outcomes.

Results: The APACHE II median score was 11 points (IQR 9-14) while 15 patients (11.5\%) died. Mortality was lower in men (10.3\%) than in women (14\%) $(P=0.04)$. APACHE II was higher in non-survivors (15; IQR 10.25-19.5) compared to survivors $(11 ; 8.25-14)(P<0.001)$. In a bivariate analysis, APACHE II predicted mortality with good diagnostic accuracy (AUROC $=0.714 ; P=0.007)$. In a multivariate analysis APACHE II (OR $=1.22 ; 95 \% \mathrm{Cl}$ : $1.06-1.40$ per 1 point) and mode of ventilation ( $\mathrm{OR}=0.28 ; 95 \% \mathrm{Cl}: 0.08-0.99 ; \mathrm{CPAP}$ vs. BIPAP) only predicted mortality. The median length of stay (LOS) was 28 days (IQR 20-39). There was weak correlation between APACHE II and LOS $(\mathrm{R}=0.23 ; P=0.01)$. Most patients were transferred to a rehabilitation center (53.9\%) or a geriatric ward (13.9\%). APACHE II did not differ between patients discharged to different healthcare facilities $(P=0.14)$.
\end{abstract}

Conclusion: The APACHE II score is a powerful tool for predicting mortality of patients undergoing weaning from prolonged mechanical ventilation.

Anaesthesiology Intensive Therapy 2016, vol. 48, no 4, 215-219

Key words: prolonged mechanical ventilation, weaning, APACHE II score, mortality, prediction

The number of patients requiring prolonged mechanical ventilation (PMV) is increasing worldwide [1]. There are multiple respiratory and non-respiratory risk factors of mechanical ventilation dependency [2]. Specialized weaning units have been established in order to facilitate the weaning of patients with continuing respiratory insufficiency who do not require an ICU level of treatment or monitoring [3]. Admission eligibility criteria usually require hemodynamic stability (no requirement for vasopressors or inotropes, no complex or life-threatening arrhythmias, no risk of acute coronary ischemia), metabolic stability, stable renal function, proper acid-base balance and the absence of an infective process [4]. Potential advantages of these facilities are the increased attention given to patient-centered rehabilitation, greater expertise and improved patient outcomes (longer survival, higher ventilator independency, better quality of life).

Mechanical ventilation predisposes patients to numerous complications having a significant impact on LOS and mortality that include pulmonary injury, pneumonia, other nosocomial infections, venous thromboembolism, dysphagia, increased catabolism with risk of malnutrition [5]. Therefore, a patient's condition on admission to a weaning unit can determine the outcome. 
The Acute Physiology and Chronic Health Evaluation II (APACHE II) score was primarily designed to measure the severity of disease in patients admitted to intensive care units (ICU) and quickly became the most popular ICU mortality prediction score. Since APACHE II was exclusively studied in patients of multidisciplinary ICUs, little is known about its diagnostic utility in patients treated in high dependency units, including weaning units.

Thus, we sought to determine the value of the APACHE II score to predict in-hospital mortality in PMV patients admitted to a regional weaning center. We also investigated the association between baseline APACHE II and LOS, as well as discharge destination in survivors.

\section{METHODS}

Our observational study included 130 consecutive patients admitted to the weaning center between 1 January 2012 and 31 December 2013. All patients had been previously hospitalized in medical/surgical intensive care units and/or high dependency postoperative care units for a median period of 22 days (IQR 15-33). Indications for admission to a weaning center were based on the guidelines from the German Respiratory Society [4]. Weaning procedures were implemented according to the national recommendations [4].

Data including demographics and comorbidities was retrieved from medical records. Clinical and laboratory data were recorded on admission in all patients. Physiological data was recorded 3-hourly on day one. The Acute Physiology and Chronic Health Evaluation II score was calculated. The calculation was based on the worst values taken during the first 24 hours post admission. The Glasgow Coma Scale (GCS) was excluded from our calculations due to a possible confounding effect from analgesic and sedative agents (baseline median RASS $=-1$ points; IQR -2 to +1) [6]. Patient confidentiality was ensured as the dataset was fully anonymized. The Ethics Committee approved the study with waiver of consent as the project was noninterventional.

In-hospital mortality was the primary outcome. LOS (days) and discharge destinations were considered secondary outcomes.

\section{STATISTICAL ANALYSIS}

A statistical analysis was performed using MedCalc Statistical Software version 14.8.1 (MedCalc Software bvba, Ostend, Belgium). Continuous variables were expressed as the median and interquartile range (IQR). Qualitative variables were expressed as a percentage. Between-group differences for quantitative variables were assessed using the Mann-Whitney U-test or the Kruskal-Wallis test. The type of distribution was verified with the Shapiro-Wilk test.
Table 1. Subjects' characteristics

\begin{tabular}{lc}
\hline Variable & $\begin{array}{c}\text { Median (IQR) } \\
\text { or percentage }\end{array}$ \\
\hline Men/Women & $66.9 \% / 33.1 \%$ \\
Age (years) & $71(62-77)$ \\
Coronary heart disease & $64.6 \%$ \\
Arterial hypertension & $92.3 \%$ \\
Previous cerebrovascular accident & $16.9 \%$ \\
Diabetes & $32.3 \%$ \\
Chronic kidney disease & $25.4 \%$ \\
Chronic obstructive pulmonary disease & $45.4 \%$ \\
Obesity (BMl > 30 kg m ${ }^{-2}$ ) & $23.1 \%$ \\
\hline
\end{tabular}

Table 2. Etiology of underlying respiratory failure

\begin{tabular}{lc}
\hline Main condition & $\%$ \\
\hline Postoperative & 58.5 \\
Pneumonia & 13.8 \\
COPD exacerbation & 5.4 \\
Post-cardiac arrest syndrome & 5.4 \\
Stroke/intracranial bleeding & 5.3 \\
Posttraumatic & 4.7 \\
Circulatory failure & 3.1 \\
Others & 3.8 \\
\hline
\end{tabular}

COPD — chronic obstructive pulmonary disease

The Mantel-Haenszel $\chi^{2}$ or Fisher's exact test were applied to qualitative variables. All tests were two-tailed. Spearman rank coefficients of correlation were calculated for appropriate quantitative data.

The impact of clinical and demographic variables on mortality was initially evaluated using a bivariate analysis. ORs with $95 \% \mathrm{Cls}$ were calculated. Variables with a $P$-value $<0.01$ were consecutively subjected to a multivariate logistic stepwise regression model. Logistic ORs with $95 \% \mathrm{Cls}$ were subsequently estimated. Receiver operating characteristic (ROC) curves were constructed and the area under the ROC curves (AUROC) was calculated to assess the predictive value of the APACHE II score on in-house mortality.

A $P$-value $<0.05$ was considered significant.

\section{RESULTS}

Patients' characteristics are shown in Table 1. The APACHE II median score (excluding GCS) was 11 points (9-14). Baseline characteristics are shown in Table 1. The most frequent admission diagnosis was postoperative respiratory failure (58.5\%) (Table 2).

Median LOS in a weaning center was 28 days (20-39). Mortality reached $11.5 \%$ (10.3\% in men, $14 \%$ in women; $P=0.04)$ and 115 patients (88.5\%) were successfully dis- 
Table 3. Discharge destination

\begin{tabular}{lc}
\hline Place of discharge & $\%$ \\
\hline Rehabilitation unit & 53.9 \\
Geriatric unit & 13.9 \\
Home & 11.3 \\
Surgical unit & 7.8 \\
Internal unit & 7.0 \\
Rest-home & 5.2 \\
Neurological unit & 0.9 \\
\hline
\end{tabular}

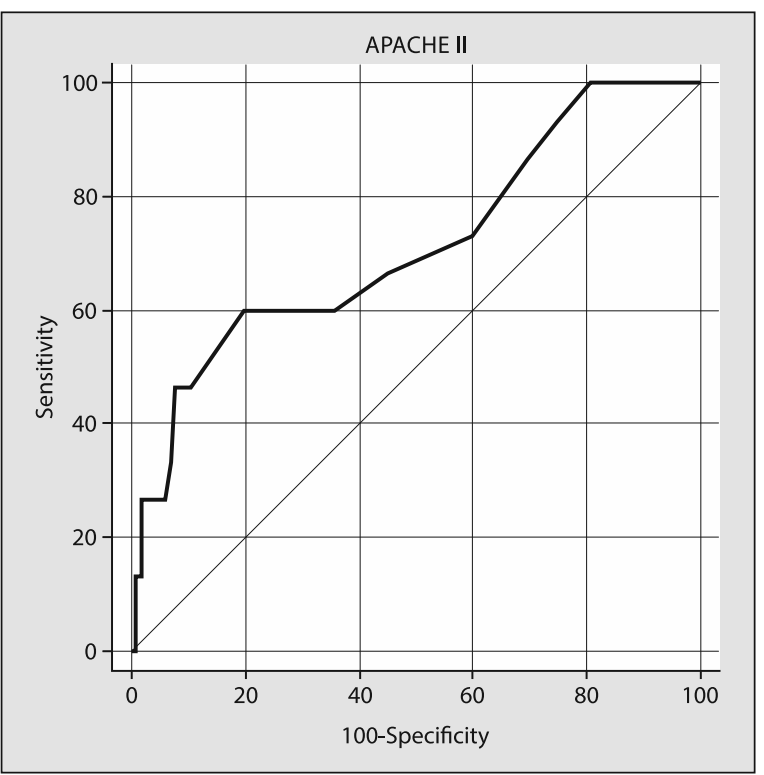

Figure 1. Receiver operating characteristic curve of APACHE II score for mortality prediction

charged (Table 3). The majority of patients were transferred to a rehabilitation center (53.9\%) or geriatric ward (13.9\%) independent of gender $(P=0.68)$.

There was weak but statistically significant correlation between APACHE II score and LOS in a weaning center $(\mathrm{R}=0.23 ; P=0.01)$. APACHE II did not differ between patients discharged to different healthcare facilities $(P=0.14)$.
The APACHE II score was statistically higher in non-survivors (median $=15$, IQR 10.25-19.5) compared to survivors (median $=11$, IQR 8.25-14) $(P<0.001)$. The APACHE II predicted mortality with good diagnostic accuracy (AUROC = $=0.714,95 \% \mathrm{Cl}: 0.628-0.798, P=0.007$ ) (Fig. 1) optimal cut-off point being 14 points (sensitivity $60 \%$; specificity $80 \%$ ).

Although by using bivariate analysis we found six predictors of mortality, a multivariate analysis revealed only the APACHE II score and mode of mechanical ventilation on admission as playing a significant role (Table 4). Mortality increased by $22 \%$ per each APACHE II point while CPAP (compared to BIPAP) decreased mortality by $72 \%$. This final 2 -variable regression equation had very good diagnostic accuracy (AUROC $=0.829 ; 95 \% \mathrm{Cl}: 0.753-0.889 ; P<0.0001$ ). The etiology of respiratory failure had no impact on mortality $(P=0.38)$.

\section{DISCUSSION}

A multidisciplinary and collaborative approach to patients improves weaning outcomes. Adequate management of PMV requires ICU protocols regarding diagnosis and treatment, as well as the systematic screening of outcomes. An individual attitude is also necessary to understand the complexity and uniqueness of treated patients. Although mortality in PMV patients is at a satisfactory level, it remains multifactorial and not fully investigated [7].

In our study we explored outcomes in ventilator-dependent patients treated in a regional weaning unit with special attention given to the association between the baseline APACHE II score and mortality. Mortality of $11.5 \%$ seems quite low and is comparable to previous studies published, conducted also in Germany $[6,8]$. Survival rates as low as $80 \%$ or less have also been reported [9]. Mortality in PMV patients is considerably high compared to simple and difficult weaning patients [10]. Damuth et al. in a systematic review and meta-analysis found that weaning outcomes were significantly worse in the USA than internationally $(P<0.001)$, with an in-hospital mortality difference of $13 \%$ (i.e. 18 vs. $31 \%$ ) and 1 -year mortality difference of $26 \%$ (i.e. 47 vs. $73 \%)$ [11].

The median APACHE II score in our patients was 11 points. Although this is comparable to some previous

Table 4. Statistically significant predictors of in-hospital mortality. Data given as ORs with $95 \% \mathrm{Cls}$

\begin{tabular}{lcc}
\hline Variable & Bivariate analysis & Multivariate analysis \\
\hline APACHE II (per point) & $1.22(1.08-1.39)$ & $1.22(1.06-1.40)$ \\
Gender (Women vs. Men) & $3.03(1.15-7.98)$ & $5.5(1.17-25.9)$ \\
Pulmonary embolism (Yes = 1) & $3.33(1.001-11.1)$ \\
Neoplastic disease (Yes = 1) & $42.78(1.95-938.54)$ & $0.28(0.08-0.99)$ \\
Dysphagia (Yes = 1) & $0.24(0.08-0.71)$ & \\
Ventilation mode (CPAP vs. BIPAP)
\end{tabular}


findings, it differs from most published data regarding PMV patients in weaning or ICU units $[2,9,12]$. There are 3 main reasons for this discrepancy. Firstly, the clinical profile of patients is changing, especially in terms of comorbidities and age [13]. Secondly, we excluded the GCS score from our calculations. Several studies have confirmed that although one's level of consciousness and mental status may influence the weaning outcome, this occurs only when they are unimpeded by analgesics and sedatives [6]. It was also confirmed that the GCS score cannot be used as a predictor of weaning outcomes [14].

The APACHE score is comprehensive and includes multiple abnormalities and chronic conditions that correspond to health status and ICU outcome. In our study, APACHE II, using the cut-off point of 14 points, predicted survival with good overall accuracy. The results are similar to the findings of Sapijaszko et al. [2] who showed that the APACHE II score was associated with mortality in PMV subjects. Sudarsanam et al. also found that mortality increased by $80 \%$ per 5 -point increase in the APACHE II score in MV patients in India [15]. The APACHE II was useful in predicting mortality in pulmonary ICU patients with a cut-off point of 16 points (AUROC = $=0.81$ ) [16]. In a comparison between tracheostomized and translaryngeally intubated patients, those with an APACHE II score $<18$ points were more likely to be successfully weaned and be kept alive [17]. Jubran et al. confirmed that the comorbidity score might increase mortality by $24 \%$ [18]. Finally, APACHE II was found to be a better predictor of mortality compared to SAPS II in patients with respiratory disease in ICUs (AUCOC $=0.81$ ) [19], as well as having a higher diagnostic accuracy compared to APACHE III and SAPS II in German interdisciplinary ICUs [20].

Contrary to the results of some authors we did not find an association between the etiology of respiratory failure and survival [8]. However, in our analysis the baseline mode of ventilation independently predicted the outcome.

\section{STUDY LIMITATIONS}

Our study possesses some limitations that prevent generalization of the obtained results. Firstly, this is a singlecenter study. A comprehensive review of the available data regarding patients requiring PMV suggests substantial variations across weaning units in terms of baseline characteristics, admission criteria and definitions used to describe PMV, as well as the diagnostic approach and treatment strategies during hospitalization [11]. Secondly, we performed a retrospective study, which may lead to researcher bias. However, we attempted to mitigate this bias by including consecutive patients during a specified time period. Thirdly, as the APACHE II includes only 14 variables, it is possible that additional unmeasured parameters may become significant predictors of mortality. However, the AUROC of our final statistical model was 0.829 and confirms good diagnostic accuracy. Finally, we used a baseline APACHE II score for prediction. Therefore, we had no insight into trends in scoring during hospitalization (i.e. fluctuation of patients' health status). Last but not least, we did not collect follow-up data regarding survival.

\section{CONCLUSIONS}

The APACHE II score is a powerful tool for predicting mortality in patients requiring prolonged mechanical ventilation treated in a weaning unit. However, the APACHE II is not useful in order to predict length of stay or discharge destination.

\section{ACKNOWLEDGEMENTS}

1. Source of funding: none.

2. Conflict of interest: none.

\section{References:}

1. Zilberberg MD, de Wit M, Shorr AF: Accuracy of previous estimates for adult prolonged acute mechanical ventilation volume in 2020: update using 2000-2008 data. Crit Care Med 2012; 40: 18-20. doi: 10.1097/ CCM.0b013e31822e9ffd.

2. Sapijaszko MJ, Brant $R$, Sandham D, Berthiaume $Y$ : Nonrespiratory predictor of mechanical ventilation dependency in intensive care unit patients. Crit Care Med 1996; 24: 601-607.

3. Burns $K E$, Lellouche $F$, Loisel $F$ et al.: Weaning critically ill adults from invasive mechanical ventilation: a national survey. Can J Anaesth 2009; 56: 567-576. doi: 10.1007/s12630-009-9124-8.

4. Boles JM, Bion J, Connors $A$ et al.: Weaning from mechanical ventilation. Eur Respir J 2007; 29: 1033-1056.

5. Chatila WM, Criner GJ: Complications of long-term mechanical ventilation. Respir Care Clin N Am 2002; 8: 631-647.

6. Barchfeld T, Dellweg D, Böckling $S$ et al.: Weaning from long-term mechanical ventilation: data of a single weaning center from 2007 to 2011. Dtsch Med Wochenschr 2014; 139: 527-533.

7. Perren A, Brochard L: Managing the apparent and hidden difficulties of weaning from mechanical ventilation. Intensive Care Med 2013; 39: 1885-1895. doi: 10.1007/s00134-013-3014-9.

8. Hannan LM, Tan S, Hopkinson Ket al.: Inpatient and long-term outcomes of individuals admitted for weaning from mechanical ventilation at a specialized ventilation weaning unit. Respirology 2013; 18: 154-160. doi: 10.1111/j.1440-1843.2012.02266.x.

9. Funk GC, Anders S, Breyer MK et al.: Incidence and outcome of weaning from mechanical ventilation according to new categories. Eur Respir J 2010; 35: 88-94. doi: 10.1183/09031936.00056909.

10. Pu L, Zhu B, Jiang L et al.: Weaning critically ill patients from mechanical ventilation: A prospective cohort study. J Crit Care 2015; 30: 862.e7-13. doi: 10.1016/j.jcrc.2015.04.001.

11. Damuth E, Mitchell JA, Bartock JL, Roberts BW, Trzeciak S: Long-term survival of critically ill patients treated with prolonged mechanical ventilation: a systematic review and meta-analysis. Lancet Respir Med 2015; 3: 544-553. doi: 10.1016/S2213-2600(15)00150-2.

12. Lone NI, Walsh TS: Prolonged mechanical ventilation in critically ill patients: epidemiology, outcomes and modelling the potential cost consequences of establishing a regional weaning unit. Crit Care 2011; 15: R102. doi: 10.1186/cc10117.

13. Carson SS, Cox CE, Holmes GM, Howard A, Carey TS: The changing epidemiology of mechanical ventilation: a population-based study. J Intensive Care Med 2006; 21: 173-182.

14. KohWY,LewTW, Chin NM, WongMF:Tracheostomy in a neuro-intensive care setting: indications and timing. Anaesth Intensive Care 1997; 25:365-368.

15. Sudarsanam TD, Jeyaseelan $L$, Thomas $K$, John G: Predictors of mortality in mechanically ventilated patients. Postgrad Med J 2005; 81: 780-783.

16. Gursel G, Demirtas S: Value of APACHE II, SOFA and CPIS scores in predicting prognosis in patients with ventilator-associated pneumonia. Respiration 2006; 73: 503-508. 
17. Wu YK, Tsai $Y H$, Lan CC et al.: Prolonged mechanical ventilation in a respiratory-care setting: a comparison of outcome between tracheostomized and translaryngeal intubated patients. Crit Care 2010; 14: R26. doi: $10.1186 /$ cc8890

18. Jubran A, Lawm G, Kelly J et al.: Depressive disorders during weaning from prolonged mechanical ventilation. Intensive Care Med 2010; 36: 828-835. doi: 10.1007/s00134-010-1842-4.

19. Del Bufalo C, Morelli A, Bassein L et al.: Severity scores in respiratory intensive care: APACHE II predicted mortality better than SAPS II. Respir Care 1995; 40: 1042-1047.

20. Markgraf R, Deutschinoff G, Pientka L, Scholten T: Comparison of acute physiology and chronic health evaluations II and III and simplified acute physiology score II: a prospective cohort study evaluating these methods to predict outcome in a German interdisciplinary intensive care unit. Crit Care Med 2000; 28: 26-33.
Corresponding author:

Łukasz J. Krzych, MD, PhD

Dept. of Cardiac Anesthesiology Intensive Care

Silesian Centre for Heart Diseases

M. Curie-Skłodowska 9, 41-800 Zabrze, Poland

e-mail: Ikrzych@sum.edu.pl

Received: 14.05.2016

Accepted: 6.06.2016 